\title{
Real-time monitoring of wood cladding spray painting properties and nozzle condition using acoustic chemometrics
}

\author{
Maths Halstensen ${ }^{1}$ Onyinye Victoria Agu ${ }^{1} \quad$ Ulrich Hundhausen $^{2} \quad$ Kim H. Esbensen ${ }^{3}$ \\ ${ }^{1}$ Department of Electrical Engineering, IT and Cybernetics, University of South-Eastern Norway, \\ Norway, maths.halstensen@usn.no \\ ${ }^{2}$ Norwegian Institute of Wood Technology (NTI), Oslo, Norway, uhunetreteknisk.no \\ ${ }^{3}$ KHE Consulting, Copenhagen, Denmark, khe.consultegmail.com
}

\begin{abstract}
An experimental setup simulating real-time wood cladding coating monitoring of nozzle conditions and spray paint properties has been investigated. This approach is based on affixed nozzle accelerometer sensors with appropriate signal conditioning and chemometric data analysis (PCA). The aim was to develop effective visualization of different process states using PCA score plots. The present feasibility study shows that this approach can be used as a basis for further development towards a Process Analytical Technology (PAT) spray monitoring system able to work in the harsh environment of an industrial wood cladding paint box. However, there is still a significant amount of on-site industrial calibration and R\&D necessary before a final method validation can be executed. The present results rely on permanently affixed PAT sensors. Further studies will a.o. focus on the degree to which replacement of acoustic accelerometer sensors necessitates recalibration of the multivariate data models employed, which is a critical success factor in industrial implementations.
\end{abstract}

Keywords: process analytical technology, PAT, multivariate data analysis, accelerometer, principal component analysis, process monitoring, nozzle spray condition, statistical quality control

\section{Introduction}

Wood has been the common façade material in Scandinavia for centuries and industrial application of exterior coatings has been markedly increasing. While quality control in the cladding industry is still mainly carried out manually, automated in-line measurements are on the rise to secure greater consistency and improve quality and productivity (Hundhausen et al., 2016).

Development of an on- and in-line quality control system at one of Europe's largest coating plants for cladding is addressed in an ongoing $R \& D$ project termed "KonTre" (Kontre). By analysis of data from many sensors located at critical stages in the full production line, necessary input/output data can be obtained for multivariate calibration and model predictions, which are essential for continuous process and product optimization. The control of the coating application is of overall importance as the film thickness is positively correlated to the product quality in terms of the coating's service life and, thus, the maintenance interval of a building façade (Grüll et al., 2014).

Regarding process yield, an instantaneous detection of spraying defects is crucial particularly in view of high feed speeds of up to $200 \mathrm{~m} / \mathrm{min}$ in industrial coating of cladding (Hundhausen et al., 2018). While abrupt failures like full clogging are easy to detect, gradual changes like nozzle wear, i.e. erosion of nozzle orifices, are not.

Acoustic chemometrics (Halstensen et al., 1998; 2000; 2001; 2006; 2010; Esbensen et al., 1998; 1999; Bakeev et al., 2010; Ihunegbo et al., 2012; Arvoh et al., 2012; Wagner et al., 2013) has proven to be a powerful tool for monitoring nozzles in various applications, such as characterization of liquid flow through an orifice plate nozzle. Also monitoring viscosity of anti-icing fluid used on aircrafts in below zero conditions has shown promising results (Halstensen et al., 2019). Its usability in wood coating processes has however not been investigated yet.

The present study aimed to assess the feasibility of using an acoustic chemometrics approach to monitor the spray nozzle performance in industrial coating of wood cladding. This was done by addressing following research questions:

1) Will the acoustic chemometrics approach, which includes Principal Component Analysis (PCA) (Esbensen and Swarbrick, 2018) and eventually Partial Least Squares regression (PLS-R), be able to extract useful information about nozzle orifice wear and clogging?

2) Will the signal from the sensor still be representative for the spraying nozzle performance or need recalibration after replacing a worn-out nozzle after typically $\sim 10001$ of paint spraying?

3) Will potential build-up of paint on the spraying nozzle and sensor affect the measurement adversely?

4) Will the method be sensitive to the paint type or can a global multivariate model be used to monitor the process independently of the paint type? 


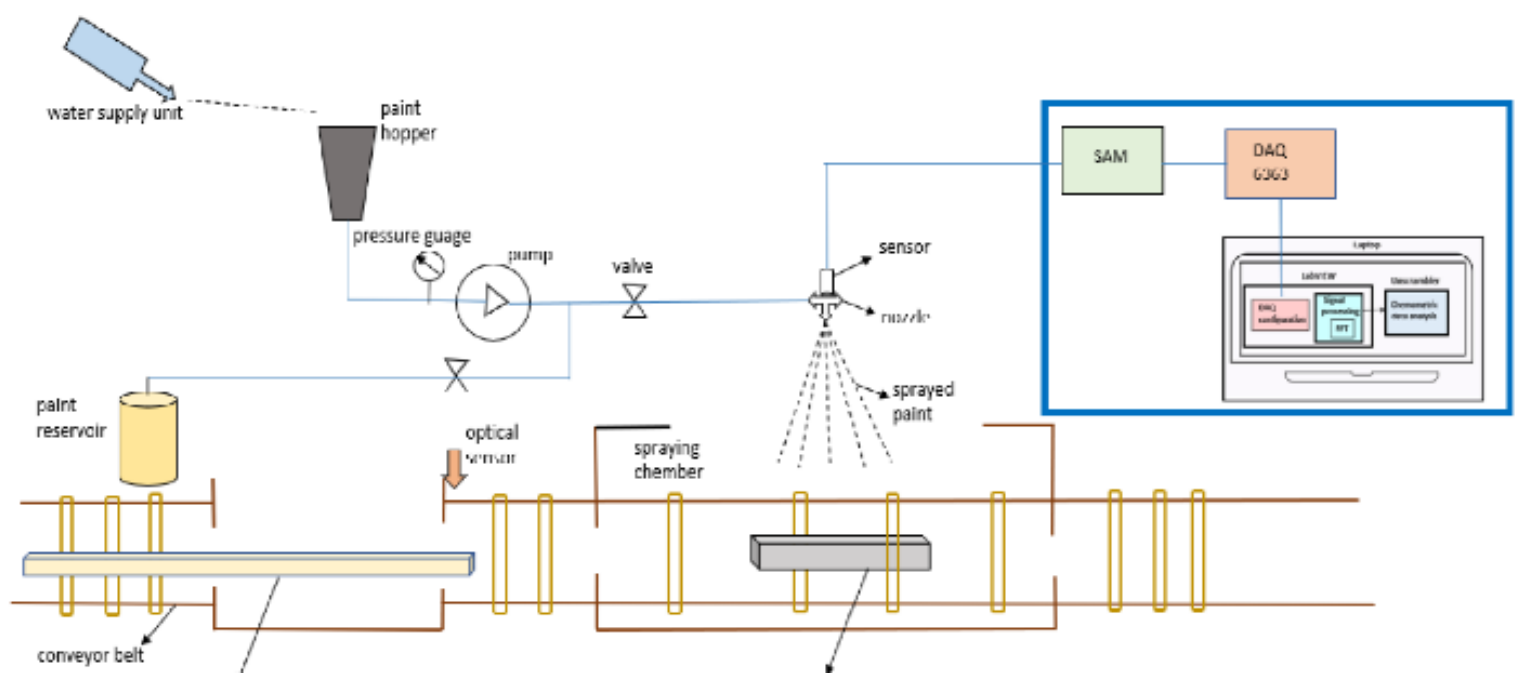

Figure 1. Simplified illustration of the experimental spraying test rig equipped with an acoustic PAT-sensor.

\section{Materials \& methods}

\subsection{Equipment}

Industrial conditions were simulated using an experimental coating line in the laboratory of the coating manufacturer Jotun AS in Sandefjord, Norway (Figure 1). The test rig consisted of a conveyer belt for the wood, an optical sensor that activates spraying, a spray chamber with an airless spraying nozzle, a water supply unit, paint hopper, pressure gauge, pump, paint mixer, paint cooling unit, hose pipe, and a handheld thermometer. The installed PAT hardware was an acoustic sensor (accelerometer) Bruel and Kjær model 4519, Signal Amplifier Module (SAM) developed at the University of South-Eastern Norway, a data acquisition device USB-6363 from National Instruments and a standard laptop computer. The software used was LabVIEW (version 2019, NI, Austin, Texas, USA), Unscrambler X (version 10.3, Camo Analytics AS, Oslo, Norway).

\subsection{Experimental design}

A full factorial design (Esbensen and Swarbrick, 2018) was employed comprising 64 experiments:

$h^{n} \cdot a=n$

where $h$ (levels) $=2, n$ (factors) $=4, a$ (paints) $=4$ and $\mathrm{n}=$ the number of experiments.

The four different paints from Jotun AS (Sandefjord, Norway) were water emulsions based on two different binders, Jotun Industri Opaque Primer and Jotun Industri Grunning Visir, and the topcoat Jotun Industri
Optimal in two different bases (

Table 1). The paints of Base A are bright due to a high content of titanium dioxide $\left(\mathrm{TiO}_{2}\right)$, those of Base $\mathrm{C}$ are dark.

Table 1. The four different paints used in the experimental design.

\begin{tabular}{ccccccc}
\hline $\begin{array}{c}\text { Paint } \\
\text { Name }\end{array}$ & Type & Binder & Base & $\begin{array}{c}\text { Titanium } \\
\text { content } \\
{[\%]}\end{array}$ & $\begin{array}{c}\text { Solid } \\
\text { content } \\
{[\mathbf{v o l \% ]}}\end{array}$ & $\begin{array}{c}\text { Specific } \\
\text { gravity }\end{array}$ \\
\hline Opaque(1) & Primer & alkyd & $\mathrm{A}$ & 16 & $46 \pm 2$ & 1.45 \\
Visir(2) & Primer & alkyd & $\mathrm{C}$ & 0 & $36 \pm 2$ & 1.04 \\
Optimal(3) & Topcoat & acrylic & $\mathrm{A}$ & 16 & $40 \pm 2$ & 1.25 \\
Optimal(4) & Topcoat & acrylic & $\mathrm{C}$ & 0 & $40 \pm 2$ & 1.25 \\
\hline
\end{tabular}

The four factors were 1) paint temperature, 2) pump pressure (paint flowrate), 3) nozzle size, and 4) nozzle condition in terms of partly clogged or free flow.

The influence of the paint temperature on the acoustic signal was tested on two levels (Table 2). The temperature levels were measured with a handheld infrared thermometer.

Table 2. Levels for the factor temperature of the four different paints in

Table 1.

\begin{tabular}{ccc}
\hline Paint type & High & Low \\
\hline Paint 1 & $21^{\circ} \mathrm{C}$ & $8{ }^{\circ} \mathrm{C}$ \\
Paint 2 & $20.8{ }^{\circ} \mathrm{C}$ & $9.6^{\circ} \mathrm{C}$ \\
Paint 3 & $20^{\circ} \mathrm{C}$ & $7.1^{\circ} \mathrm{C}$ \\
Paint 4 & $20^{\circ} \mathrm{C}$ & $7.5^{\circ} \mathrm{C}$ \\
\hline
\end{tabular}

The specific pump pressures (factor 2) applied for each paint were 0.4 and 0.3 bar. The orifice diameter of the nozzles (factor 3) were $0.025 \mathrm{inch}(0.635 \mathrm{~mm})$ and 0.019 inch $(0.483 \mathrm{~mm})$, respectively. The two nozzle sizes (factor 3 ) were tested under clogged and open conditions while other variables remained constant (high and low temperature, high and low pressure). Both nozzles had a spraying angle of $60^{\circ}$. 
The influence of the nozzle condition (factor 4) on the acoustic signal was studied on nozzles with a free flow and nozzles that were partly clogged. The latter was induced by forcefully inserting a wedge-shaped wood splinter in the nozzle opening (Figure 2). As this was done manually, the clogging conditions were not identical in all experiments. In the eleventh measurement, the clogging material fell out towards the last part of the experiment, conveniently simulating a transient clogging situation.

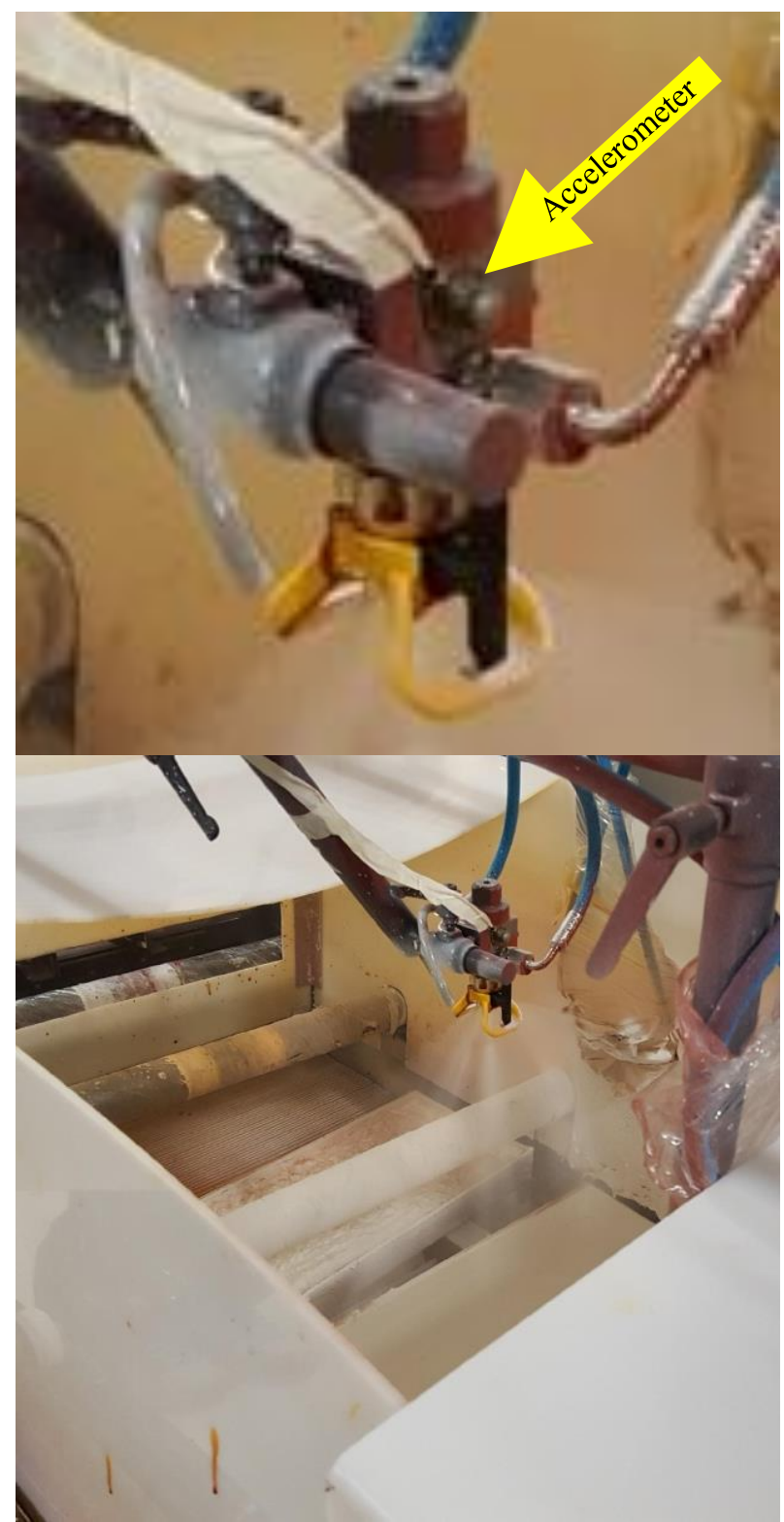

Figure 2. Upper: Spraying nozzle assembly with accelerometer. Lower: Disturbed spray pattern from a partly clogged nozzle.

Each paint type was mixed thoroughly in the paint mixer to achieve homogeneity. The paint was transferred to the hopper, ready to be pumped through the rig (Figure 1). Before applying a new paint (Table 1), hosepipes a.o. parts were rinsed with high-pressure water.
The temperature of the paint was measured manually with a handheld thermometer before it was pumped into the hopper in the test rig.

Once the optical sensor mounted on the conveyor belt detected a wood board, the nozzle started to spray paint, and the acoustic sensor started to capture the measurement signal in each test, 100 acoustic spectra were captured and then converted to one spectrum to get a precise measurement. This was repeated 5 times to give 5 replicates, which was saved on the computer. At the end of each experimental run, the used paint was transferred into the waste container.

\subsection{Acoustic chemometrics}

Figure 3 shows the acoustic chemometric signal path involved in acquiring vibrational signals from the active nozzle and their subsequent signal processing.
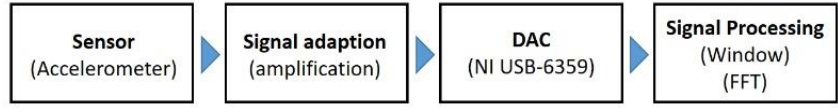

Figure 3. Block diagram of processes involved in acquiring vibrational signal from active spraying nozzles.

The acoustic sensor used is a piezoelectric shear accelerometer type 4518. In general, this type of PAT sensor is used for vibration monitoring, modal and structural analyses. Four different piezoelectric accelerometers were used for different experimental tests, which are discussed in the later sections. The piezoelectric sensor generates an electric charge signal proportional to the vibration acceleration due to the flow of paint through the nozzle bore and its sudden depressurized spraying action upon leaving the bore orifice.

The electric signal is produced when the inbuilt crystal material is subjected to a shear force. When the mass attached on the crystal material experiences a vibration, it causes the piezoelectric crystal to stretch and rebound, thereby generating opposite charges that are transmitted as an electric signal. The piezoelectric sensor has an inbuilt amplifier that converts the output charges into a low-impedance voltage signal between 1 and $100 \mathrm{mV}$. The sensor has a high resistance to base bending and a high response frequency range $>20 \mathrm{~Hz}$, and can also withstand temperature changes between 51 and $121^{\circ} \mathrm{C}$. Its low impedance output characteristic leads to low operating voltage, making it more economical and safer for use. It has low resistivity, i.e. any small signal can be detected and transmitted. Its output impedance $<100 \Omega$. The other end of the sensor cable was connected to the input channel port on the Signal Amplifier Module (SAM).

The SAM amplifies the signal from the acoustic sensor $(1-100 \mathrm{mV})$ in order to use the entire input range which is $\pm 10 \mathrm{~V}$. Having a large signal range will guarantee maximum digital resolution in the following 
analog-to-digital converter. When the signal is amplified, the signal amplitude obtained is about $\pm 5 \mathrm{~V}$, which will then flow to the DAQ unit.

The USB 6363 data acquisition (DAQ) data acquisition hardware is used to acquire a signal (like analog, digital, etc.) from a sensor into a laptop. It is capable of handling analog to digital signal conversion, A/D. Since the signal from SAM is accessed in the computer, the SAM signals must be converted from analog to digital format.

The DAQ 6363 has a 16 bits resolution (i.e. the smallest change the unit can measure). Its maximum sampling frequency (how fast sampling is done) rate is $2 \mathrm{MHz}$. The vibration frequency is between $0-100 \mathrm{kHz}$. Thus, the sampling rate used in this work is $200 \mathrm{kHz}$ to satisfy Shannon's sampling theorem. The configuration of this unit was carried out in LabVIEW.

The most important signal processing steps involved is shown in Figure 4, where a time series of 4096 samples corresponding to $0.02 \mathrm{sec}$ is recorded from the accelerometer. The time series is then multiplied with a Blackman Harris window (Ifeachor and Jervis 1993) canceling out the signal towards the ends of the series to prevent spectral leakage. The final step is the Discrete Fourier Transform (DFT) that is used to transform the time domain signal into the frequency domain. The DFT can be expressed as

$X_{k}=\sum_{n=0}^{N-1} x_{n} e^{-i 2 \pi k n / N} \quad k=0, \ldots, N-1$

A more efficient implementation of the DFT is the Fast Fourier Transform (FFT) (Ifeachor and Jervis, 1993) which in this work has been implemented in LabVIEW 2017 for fast real time calculation of the Fourier spectrum. Figure 4 shows a part of the LabVIEW GUI where the time series (4096 samples) is shown on top, and the FFT spectrum below. This spectrum represents the vibration signal in the pertinent time interval; it can be viewed as a "spectral fingerprint" of the state of the Normal Operating Conditions (NOC) of the nozzle while at work.

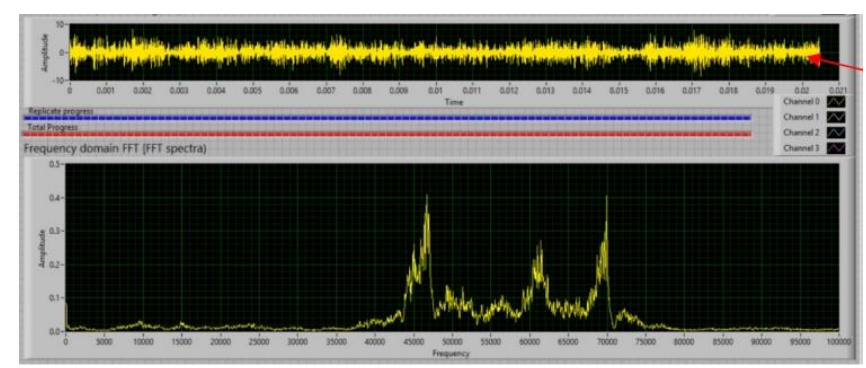

Figure 4. Top: time domain signal indicated with a red arrow, Bottom: derived frequency domain (FFT) spectrum. This spectral fingerprint is used directly in the multivariate calibration of a state prediction model (see text).

\subsection{Principal component analysis}

Principal component analysis (PCA) is a standard multivariate data analysis method (Esbensen and Swarbrick, 2018; Martens and Næs, 1989). It uses an orthogonal transformation to convert a set of observations often described by many correlated variables into a few linearly uncorrelated latent variables called principal components. We used the chemometric iterative NIPALS algorithm. Principal component analysis can alternatively be carried out based on singular value decomposition (SVD). The advantage of using SVD is that the code is simple, the disadvantage is that all possible components are always calculated, i.e., if $\mathrm{X}$ is large (i,e, is made up of a high number of variables), SVD is time consuming. NIPALS is slightly more complex to program, but a major advantage is that it allows to define the number of components to calculate. Thus, it saves time if $\mathrm{X}$ has many samples and variables. NIPALS also works on matrices with missing values; in that case SVD will fail. NIPALS is the standard chemometric approach and has been described in full detail in a wealth of literature (Esbensen and Swarbrick, 2018; Martens and Næs, 1989).

A series of spectral fingerprints (observations) can be assembled into an X-matrix with the 2048 FFT spectral frequencies as variables.

\section{Results \& discussion}

Below follows the relevant PCA score-plots delineating the degree of discriminability obtained from running experiments pertaining to various factors and levels. In all the score plots, the average acoustic spectrum is shown to have a common reference point for all the score plots. For simplicity, only the results for paint 1 are shown for the investigations on the effect of the paint temperature, pressure and nozzle state (new, worn, clogged). The other paint types show similar results.

\subsection{The effect of paint temperature}

Figure 5 shows the score plot $\mathrm{t} 1 \mathrm{t} \mathrm{t} 2$ of the acoustic measurement at different temperatures. The baseline is indicated as "Average" while the low and high temperatures applied in the different groups of samples are labelled "Low Temp" and "High Temp", respectively. The groups are clearly separated, showing that the nozzle vibrations depend on the temperature. Both groups of samples lie to the right side of the base group, which suggests that the amplitude increases due to the flow through the nozzle. PC2 holds the information of the two sample groups with different temperature as their separation is mainly in the PC2 direction. 

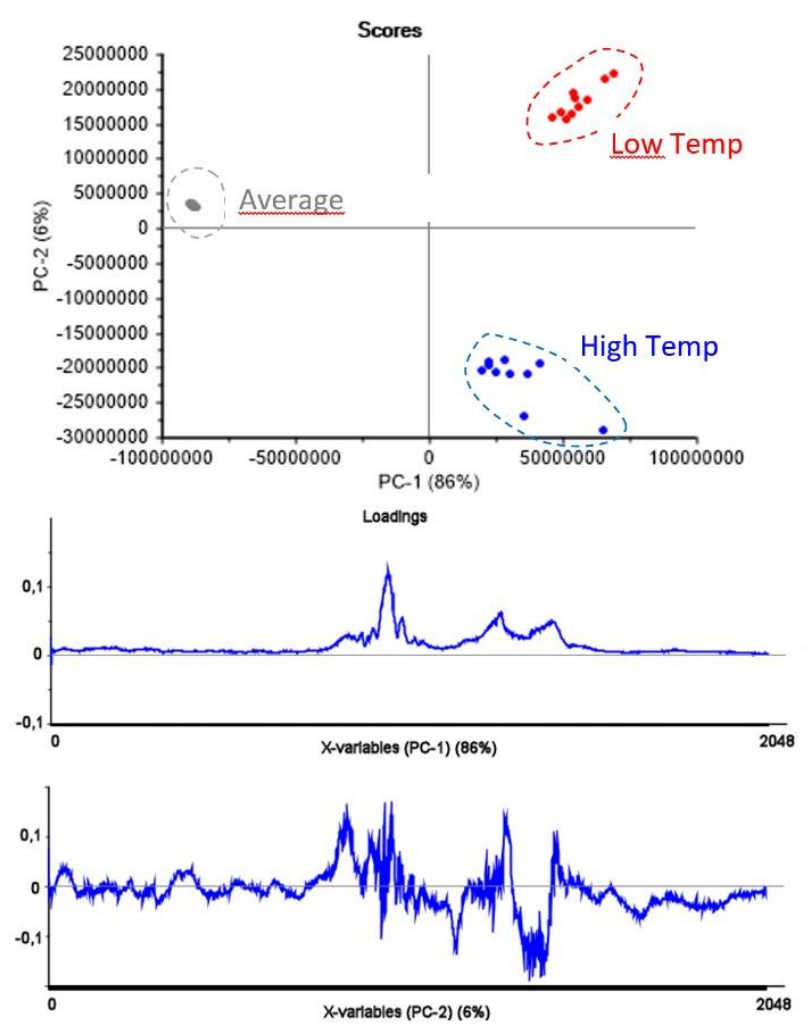

Figure 5. The effect of a temperature change, from top to bottom: Scores t1-t2, Loading 1, Loading 2

\subsection{The effect of pump pressure}

Figure 6 shows the score plot $\mathrm{t} 1-\mathrm{t} 2$ of the acoustic measurement at two different spraying pressures (high/low). Like for the effect of paint temperature, the baseline is also here indicated as "Average" while the low and high pressures are labelled "Low Pressure" and "High Pressure", respectively.

The groups are clearly separated, which indicates that the nozzle vibration depends on the paint pressure. Like in the score plot for the paint temperature, both groups of samples lie to the right side of the base group, indicating an increase in the amplitude due to the flow through the nozzle. PC2 holds the information of the two sample groups with different pressure as their separation is mainly in the PC2 direction.

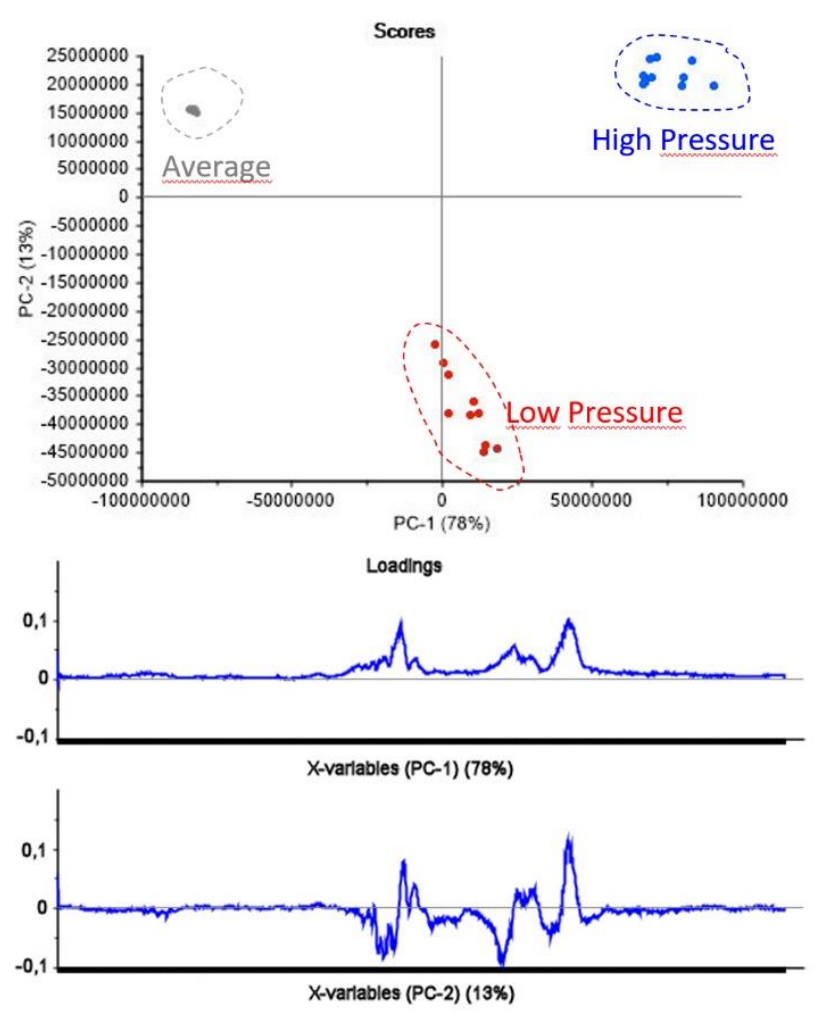

Figure 6. The effect of a change in pressure, from top to bottom: Scores t1-t2, Loading 1, Loading 2

\subsection{The effect of nozzle wear}

It is of high interest to monitor the state of a spraying nozzle as a worn nozzle changes the spray pattern. This can increase the loss of paint due to overspray and/or cause spraying defects. A new nozzle will typically last for 800 to 1500 liters of paint depending on spraying conditions and the paint type.

In Figure 7, two different nozzle conditions (new and worn) are shown in the score plot $\mathrm{t} 1-\mathrm{t} 2$ of the acoustic measurements. Like in Figure 5 and 6, the baseline is indicated as "Average" while the condition of the nozzle is indicated "new" and "worn".

The different location of the groups in the score plot indicates that the nozzle vibration depends on nozzle wear. The results are promising for the possibility of online monitoring of the nozzle state. A new nozzle will start with a negative score in the PC2 direction and slowly move to the direction of a positive score. 

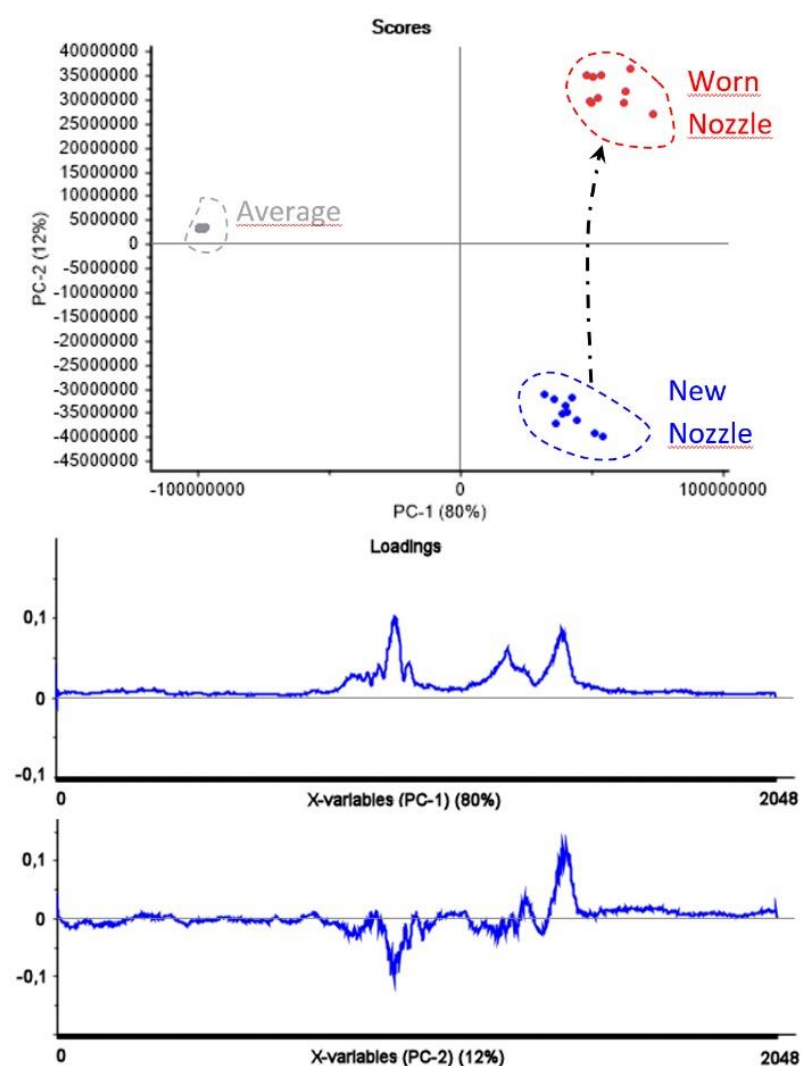

Figure 7. The effect of a nozzle wearing out, from top to bottom: Scores t1-t2, Loading 1, Loading 2

\subsection{The effect of clogging}

Another important aspect of process monitoring is the immediate detection of nozzle clogging because it causes coating defects and thus production loss. Figure 8 shows the results of a new and a worn nozzle in clogged and unclogged condition. Like in Fig. 7, the scores of a new and a worn nozzle in normal operation are clearly distinguishable from each other. The difference between scores of a clogged and an unclogged nozzle is even larger and allows an easy detection.

From these PCA results, there is a clear indication that the acoustic chemometric approach present the critical information needed to monitor the quality of paint spray evenness and nozzle condition during wood cladding.

\subsection{The effect of paint type}

Figure 9 shows the score plot $\mathrm{t} 1-\mathrm{t} 2$ of all four paint types, all the other variables such as temperature and pressure were kept constant. The score plot results suggest a clear influence of the paint type on the nozzle vibration. Paint 1 has the highest score along PC1, which indicates that this paint produces the highest sound amplitude. This can be probably ascribed to the high solid content and specific gravity of paint type 1 of 46 vol\% and 1.45 , respectively (Table 1 ).
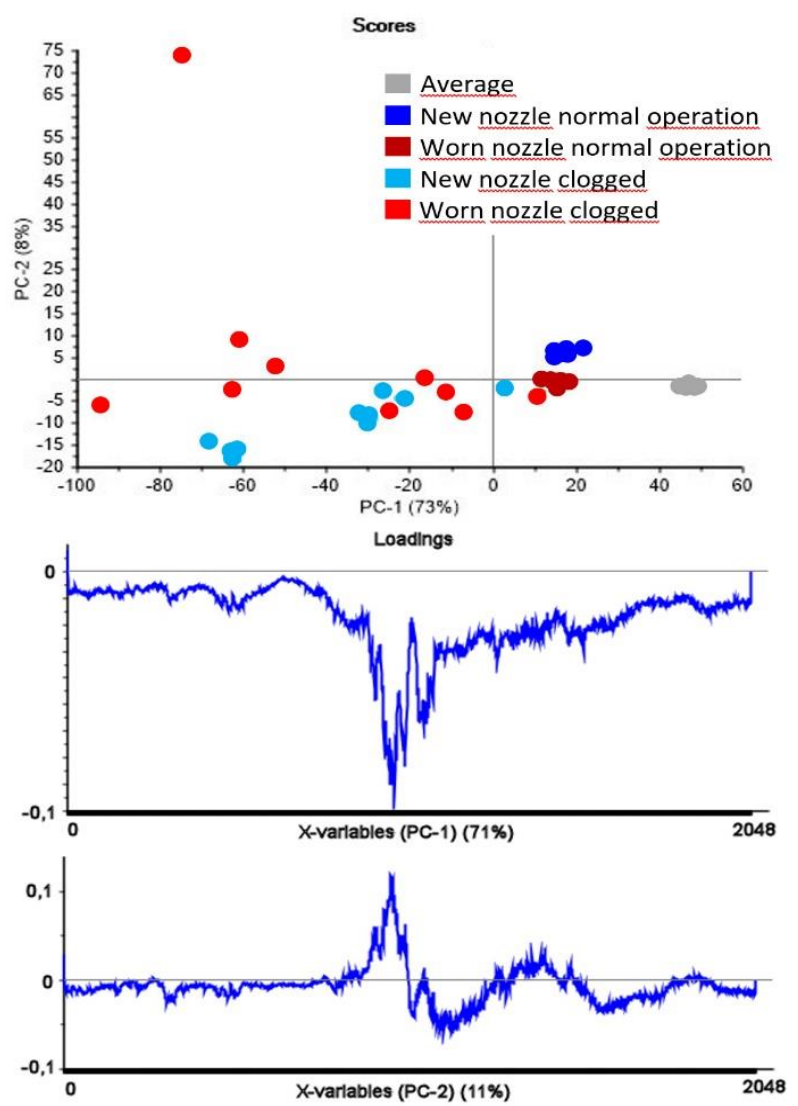

Figure 8. The effect of a clogged paint nozzle, from top to bottom Scores t1-t2, Loading 1, Loading 2
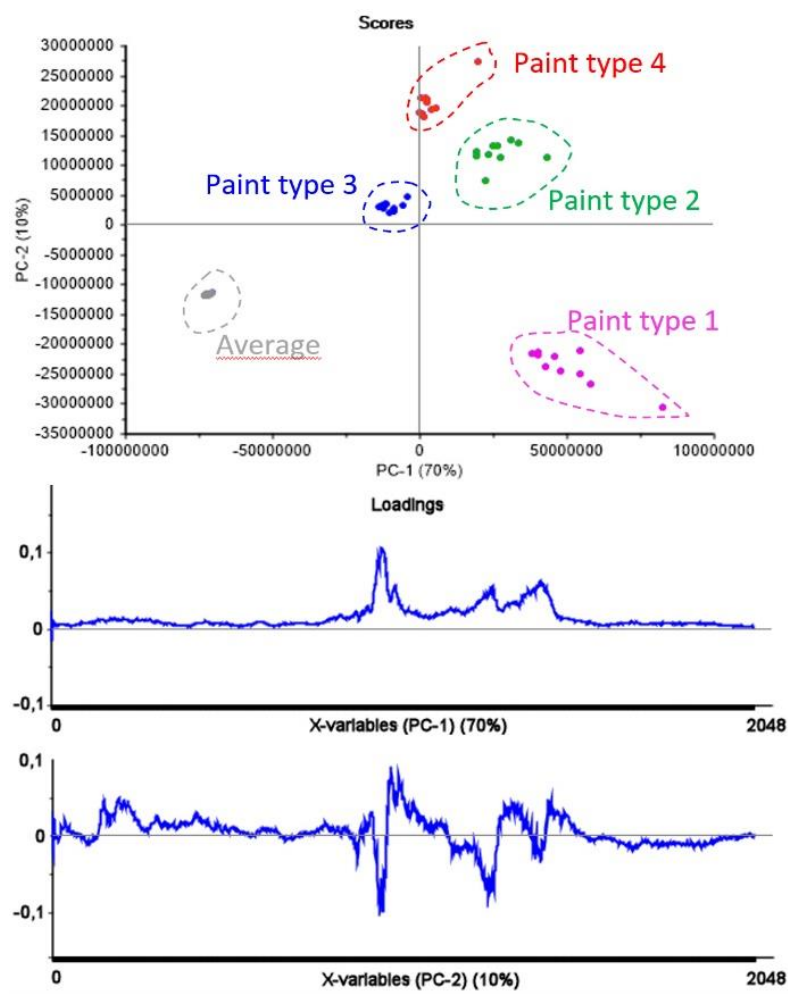

Figure 9. The effect of changing paint type, from top to bottom Scores t1-t2, Loading 1, Loading 2 


\section{Conclusion}

The experimental setup based on accelerometer sensors with appropriate signal conditioning a.o. allows effective visualization of the different process states for different paints and working conditions using PCA score plots.

The present feasibility study shows the potential of our acoustic chemometric approach for the development of an industrial PAT paint spray monitoring system for wood coatings. However, there is a significant amount of on-site industrial calibration R\&D necessary before a final method validation can be undertaken. The next steps will be to assess the technology in the harsh industrial environment of a coating plant for wood cladding. The present results rely on permanently affixed PAT sensors. Further studies will a.o. focus on the degree to which replacement of acoustic accelerometer sensors necessitate re-calibration of the multivariate data models employed - a critical issue for industrial implementation of acoustic chemometrics.

Finally, the applicability of acoustic chemometrics to other coating types and finishing processes than cladding, such as furniture, windows or flooring should be assessed.

\section{Acknowledgements}

This study was funded by The Research Council of Norway as part of the project KonTre (Innovation Project for the Industrial Sector-BIONÆR, project no. 269206) and carried out in cooperation between NTI (Norwegian Institute of Wood Technology), the University of South-Eastern Norway, KHE Consulting, Copenhagen, Jotun AS and Gausdal

\section{References}

B. K. Arvoh, S. Asdahl, K. Rabe, R. Ergon, and M. Halstensen. Online estimation of reject gas and liquid flow rates in compact flotation units for produced water treatment. Flow Meas. Instrum., 24: 63-70, 2012. doi:10.1016/j.flowmeasinst.2012.03.008

K. A. Bakeev, Process Analytical Technology, 2nd edition. Wiley, Chichester, United Kingdom, 2010. ISBN: 978-0470-72207-7, 2010, doi:10.1002/9780470689592

K. H. Esbensen, and B. Swarbrick, Multivariate Data Analysis - An introduction to Multivariate Analysis, Process Analytical Technology and Quality by design, 6th Ed., CAMO Publishing, 2018, ISBN 078-82-691104-0-1

K. H. Esbensen, B. Hope, T. T. Lied, M. Halstensen, T. Gravermoen, and K. Sundberg. Acoustic chemometrics for fluid flow quantifications: II. A small constriction will go a long way. Journal of Chemometrics 13 (27): 209-236, 1999. doi: 10.1002/ (SICI) 1099-128X (199905/08)13:3/4b209::AID-CEM553>3.0.CO;2-5.

K. H. Esbensen, M. Halstensen, T. T. Lied, A. Saudland, J. Svalestuen, S. de Silva, and B. Hope. Acoustic chemometrics - from noise to information. Chemometr.
Intell. Lab Syst. 44 (1-2): 61-76, 1998. doi:10.1016/S01697439 (98)00114-2.

G. Grüll, F. Tscherne, I. Spitaler, and B. Forsthuber, Comparison of wood coating durability in natural weathering and artificial weathering using fluorescent UVlamps and water. European Journal of Wood and Wood Products 72(3): 367-376, 2014. doi:10.1007/s00107-0140791-y

M. Halstensen, Acoustic Chemometrics, Experimental multivariate sensor technology and development of system prototypes for industrial multi-phase characterisation: selected forays. Ph.D. thesis, Telemark University College, Porsgrunn, Norway, 2001, ISBN 82-471-5381-5, ISSN 0809-103X

M. Halstensen, J. Lundberg, P.I. Januschas, and H.-P. Halvorsen, On-line Monitoring of Viscous Properties of Anti-icing Fluid Based on Partial Least Squares Regression Modeling, Linköping Electronic Conference Proceedings, 170:26-31, 2019. 10.3384/ecp2017026

M. Halstensen and K. H. Esbensen. New developments in acoustic chemometrics prediction of particle size distribution-the problem is the solution. Journal of Chemometrics, 14: 463-481, 2000. doi:10.1002/1099128X(200009/12)14:5/6b463:: AID-CEM628>3.0.CO;2Y.

M. Halstensen and K.H. Esbensen. Acoustic chemometric monitoring of industrial production processes in: K.A. Bakeev, Process Analytical Technology, Wiley, Chichester, United Kingdom, 2: 281-302, 2010. doi:10.1002/9780470689592, ISBN: 978-0-470-72207-7

M. Halstensen, P. de Bakker, and K. H. Esbensen. Acoustic chemometric monitoring of an industrial granulation production process - a PAT feasibility study. Chemometr. Intell. Lab Syst, 84: 88-97, 2006. doi:10.1016/j.chemolab.2006.05.012

M. Halstensen, S. R. de Silva, and K.H. Esbensen. Acoustic monitoring of pneumatic transport lines-from noise to information. KONA Powder and Particle 16 (8): 170-178 1998. doi:10.14356/KONA.1998020

U. Hundhausen, D. Kraniotis, S. Charisi, and M-L. Sortland, Quality control in coating lines for exterior cladding. In: Proceedings International Woodcoatings Congress 2016. 25-26, 2016. ISBN: 9783866302815

U. Hundhausen, M. Slabohm, and P. Meinlschmidt, Industrial coating of wood cladding: Inline control of board temperature, film thickness, and microfoam. In: Proceedings International Woodcoatings Congress 2018. 20-21, 2018. ISBN: 9783748600039

Emanuel Ifeachor and Barrie Jervis, Digital Signal Processing, a Practical Approach, Addison-Wesley Publishing, 1993. ISBN-13: 978-0201596199

F. N. Ihunegbo, M. Madsen, K. H. Esbensen, J. B. HolmNielsen, and M. Halstensen. Acoustic chemometric prediction of total solids in bioslurry: A full-scale feasibility study for on-line biogas process monitoring. Chemometr. Intell. Lab. Syst., 110: 135-143, 2012. doi:10.1016/j.chemolab.2011.10.009

KonTre (Innovation Project for the Industrial SectorBIONÆR, project no. 269206) 
H. Martens and T. Næs. Multivariate Calibration, Wiley, Chichester, UK, 1989 reprint 1994. ISBN-10: 0471930474, ISBN-13: 978-0471930471

C. Wagner, F. N. Ihunegbo, M. Halstensen, and K.H. Esbensen. Acoustic chemometrics for material composition quantification in pneumatic conveying - The critical role of representative reference sampling. Powder Technology, 237: 506-513, 2013. doi:10.1016/j.powtec.2012.12.036 